\title{
A NOTE ON A PAPER BY WONG AND HEYDE
}

\author{
ALEKSANDAR MIJATOVIĆ, ${ }^{*}$ University of Warwick \\ MIKHAIL URUSOV,** Ulm University
}

\begin{abstract}
In this note we re-examine the analysis of the paper 'On the martingale property of stochastic exponentials' by Wong and Heyde (2004). Some counterexamples are presented and alternative formulations are discussed.
\end{abstract}

Keywords: Local martingales versus true martingales; stochastic exponential

2010 Mathematics Subject Classification: Primary 60G44; 60G48; 60H10; $60 \mathrm{~J} 60$

\section{Introduction}

In [5] Wong and Heyde announced very general results about the martingale property of exponential local martingales. Since the subject matter of the paper is important, it is necessary to examine it critically. In Section 2 we describe the setting of [5]. In Section 3 we re-examine the analysis in [5] and discuss an alternative formulation of their Corollary 2. Section 4 contains counterexamples.

\section{The setting in [5]}

The main object of study in [5] is the stochastic exponential

$$
\begin{aligned}
& Z_{X}(t)=\exp \left\{\int_{0}^{t} X(u) \cdot \mathrm{d} W(u)-\frac{1}{2} \int_{0}^{t}\|X(u)\|^{2} \mathrm{~d} u\right\}, \quad t \in[0, \infty), \\
& Z_{X}(0)=1,
\end{aligned}
$$

where $X(t) \in \mathbb{R}^{d}$ is a $d$-dimensional $\left(\mathscr{F}_{t}\right)$-progressively measurable process and $W$ is a $d$-dimensional Brownian motion defined on a given probability space $(\Omega, \mathcal{F}, \mathrm{P})$. The filtration $\left(\mathcal{F}_{t}\right)_{t \in[0, \infty)}$ is assumed to be generated by $W$ and augmented to satisfy the usual conditions (see the second paragraph on page 656 of [5] for these assumptions).

The stopping time $\tau^{M_{X}}$ is defined by

$$
\tau^{M_{X}}=\lim _{N \rightarrow \infty} \tau_{N}^{M_{X}}, \quad \text { where } \tau_{N}^{M_{X}}=\inf \left\{t \in[0, \infty): \int_{0}^{t}\|X(u)\|^{2} \mathrm{~d} u \geq N\right\},
$$

with the usual convention that $\inf \varnothing=\infty$ (see Equation (2) on page 656 of [5]). The nondecreasing adapted process $M_{X}$, defined by

$$
M_{X}(t):=\int_{0}^{t}\|X(u)\|^{2} \mathrm{~d} u \quad \text { for } t \in[0, \infty),
$$

Received 21 October 2010; revision received 16 May 2011.

* Postal address: Department of Statistics, University of Warwick, Coventry CV4 7AL, UK.

Email address: a.mijatovic@warwick.ac.uk

** Postal address: Institute of Mathematical Finance, Ulm University, Ulm 89081, Germany.

Email address: mikhail.urusov@uni-ulm.de 
is left continuous by the monotone convergence theorem. On the event $\left\{\tau^{M_{X}}<\infty\right\}$, at time $\tau^{M_{X}}$ the process $M_{X}$ either jumps to $\infty$ if $M_{X}\left(\tau^{M_{X}}\right)<\infty$ or tends to $\infty$ continuously (i.e. assumes arbitrarily large values just before $\left.\tau^{M_{X}}\right)$ if $M_{X}\left(\tau^{M_{X}}\right)=\infty$. A precise definition of the stochastic exponential $Z_{X}$ in (1) can now be given by

$$
Z_{X}(t):=\exp \left\{\int_{0}^{t \wedge \tau^{M_{X}}} X(u) \cdot \mathrm{d} W(u)-\frac{1}{2} \int_{0}^{t \wedge \tau^{M_{X}}}\|X(u)\|^{2} \mathrm{~d} u\right\} \quad \text { for } t \in[0, \infty),
$$

where we set $Z_{X}(t)=0$ on $\left\{\tau^{M_{X}} \leq t, M_{X}\left(\tau^{M_{X}}\right)=\infty\right\}$. Note that the stochastic integral in (3) is well defined P-almost surely (P-a.s.) on $\left\{\tau^{M_{X}}<\infty, M_{X}\left(\tau^{M_{X}}\right)<\infty\right\}$ for every $t \in$ $[0, \infty)$, and, hence, the process $Z_{X}$ is continuous and takes strictly positive values on the event $\left\{\tau^{M_{X}}=\infty\right\} \cup\left\{\tau^{M_{X}}<\infty, M_{X}\left(\tau^{M_{X}}\right)<\infty\right\}$. Furthermore, the stochastic exponential $Z_{X}$ is a continuous local martingale (the continuity at $\tau^{M_{X}}$ on the event $\left\{\tau^{M_{X}}<\infty, M_{X}\left(\tau^{M_{X}}\right)=\infty\right\}$ follows by the Dambis-Dubins-Schwarz theorem; see, e.g. [4, Chapter V, Theorem 1.6]).

On page 656 of [5], in the line following the formula containing the definition of $\tau_{N}^{M_{X}}$, the authors stipulated that their process $X$ is stopped at $\tau^{M_{X}}$, which is rather confusing because this implies that $\mathrm{P}\left(\tau^{M_{X}}<\infty, M_{X}\left(\tau^{M_{X}}\right)<\infty\right)=0$ and, thus, restricts the generality without being essential for the setting and what follows (see, however, a more precise and detailed discussion on a related point in item 5 of Section 3 below). Note that the event $\left\{\tau^{M_{X}}<\infty\right.$, $\left.M_{X}\left(\tau^{M_{X}}\right)<\infty\right\}$ can in general be the entire space $\Omega$ (e.g. take $X$ deterministic).

Finally, it should be noted that the authors of [5] worked on a finite time interval $[0, T]$ (see, e.g. the second paragraph on page 656 of [5]), while the setting introduced above is the infinite time horizon setting. This difference does not affect the exposition below but lets us quote many formulae from [5] exactly as they are stated there without introducing inconsistency. (There are notational inconsistencies in [5] related to this point: e.g. they defined $\tau_{N}^{M_{X}}$ exactly as in (2), but if one works on a finite time interval $[0, T]$ and the process $X(t)$ is given for $t \in[0, T]$, one should have used either the definition

$$
\inf \left\{t \in[0, T]: \int_{0}^{t}\|X(u)\|^{2} \mathrm{~d} u \geq N\right\}
$$

or the definition

$$
\inf \left\{t \in[0, T]: \int_{0}^{t}\|X(u)\|^{2} \mathrm{~d} u \geq N\right\} \wedge T
$$

for $\tau_{N}^{M_{X}}$.)

\section{Discussion of Section 3 of [5]}

In this section we reinspect Proposition 1 of [5], pointing to problems in its formulation and proof. This has consequences for the rest of the work in [5]. The formulation and the proof of the main result, Theorem 1 of [5], both rely on Proposition 1. Furthermore, in Definition 1 of [5] the central concept of a 'candidate measure' is introduced. It is implicitly assumed throughout [5] that the defined object exists and is unique. However, the 'candidate measure' may not in fact exist, and if it does, it may not be unique. Thus, Corollaries 1 and 2 of [5], which in their formulation use the notion of the 'candidate measure', are invalid. Corollary 2 of [5] admits an obvious well-posed reformulation, but the resulting statement, given in Corollary $2^{*}$ below, is also invalid (see Section 4 for a counterexample).

1. We first discuss Proposition 1 of [5], which plays a key role in [5] as it is used in the formulation and applied in the proof of the main result of [5] (Theorem 1). We start by stating Proposition 1 of [5, Section 3, p. 657]. 
Proposition 1. (Proposition 1 of [5].) Consider a d-dimensional $\mathcal{F}_{t}$-progressively measurable process $X(t)=\xi(W(\cdot), t)$ defined possibly up to the explosion time $\tau^{M_{X}}$ defined by (2). Then there will also exist a d-dimensional $\mathcal{F}_{t}$-progressively measurable process $Y(t)=\xi(W(\cdot)+$ $\left.\int_{0}^{\cdot} Y(u) \mathrm{d} u, t\right)$, defined possibly up to the explosion time $\tau^{M_{Y}}$, with

$$
\tau^{M_{Y}}=\lim _{N \rightarrow \infty} \tau_{N}^{M_{Y}}
$$

where

$$
M_{Y}(t)=\int_{0}^{t}\|Y(u)\|^{2} \mathrm{~d} u, \quad \tau_{N}^{M_{Y}}=\inf \left(t \in[0, \infty): \int_{0}^{t}\|Y(u)\|^{2} \mathrm{~d} u \geq N\right) .
$$

The formulation of Proposition 1 is misleading. Firstly, the formula ' $Y(t)=\xi(W(\cdot)+$ $\left.\int_{0} Y(u) \mathrm{d} u, t\right)^{\prime}$ ' cannot be a definition of $Y$. This is an equation in $Y$. Secondly, the statement in the proposition that ' $\ldots$ the process $Y(t)=\xi\left(W(\cdot)+\int_{0}^{\cdot} Y(u) \mathrm{d} u, t\right)$ defined possibly up to the explosion time $\tau^{M_{Y}} \ldots$ ' is followed by the definition of $\tau^{M_{Y}}$, which is given in terms of $Y$ that has not yet been defined.

Let us now analyse the proof of Proposition 1 of [5] in the hope that it will shed light on its formulation. The proof operates with a process $X_{N}$, which is not introduced in [5]. However, the formula ' $Z_{X}\left(t \wedge \tau^{M_{X}}\right)=Z_{X_{N}}(t)$ ' in the first line of the proof (which should read as ' $Z_{X}\left(t \wedge \tau_{N}^{M_{X}}\right)=Z_{X_{N}}(t)$ ', as supported by what follows and because the authors of [5] refered to their Lemma 1 in the second line of the proof) makes it evident that they meant $X_{N}(t)=X(t) \mathbf{1}\left(t \leq \tau_{N}^{M_{X}}\right)$. The authors of [5] defined a measure $\mathrm{Q}_{N}$, equivalent to $\mathrm{P}$, by $\mathrm{Q}_{N}(A)=\mathrm{E}_{\mathrm{P}}\left[Z_{X_{N}}(T) \mathbf{1}(A)\right]$ for all $A \in \mathcal{F}_{T}$. (Here we corrected another misprint: in [5] they wrote ' $\mathrm{Q}_{N}\left(X_{N} \in A\right)=\mathrm{E}_{\mathrm{P}}\left[Z_{X_{N}}(T) \mathbf{1}\left(X_{N} \in A\right)\right]$ for all $A \in \mathcal{F}_{T}$ '.) Furthermore, they defined a $d$-dimensional $\mathrm{Q}_{N}$-Brownian motion $W^{\mathrm{Q}_{N}}$ by the formula

$$
W^{\mathrm{Q}_{N}}(t)=W(t)-\int_{0}^{t} X_{N}(u) \mathrm{d} u .
$$

Then, in line 2 on page 658 of [5] the identity

$$
X_{N}(t)=\xi\left(W^{\mathrm{Q}_{N}}(\cdot)+\int_{0}^{\cdot} X_{N}(u) \mathrm{d} u, t\right) \quad \text { on }\left\{t \leq \tau_{N}^{M_{X}}\right\}
$$

is stated, which is correct. However, all that follows in the proof of Proposition 1 has problems. It is stated in [5] that (4) 'demonstrates the existence up to $\tau_{N}^{M_{Y}}$ of'

$$
Y_{N}(t)=\xi\left(W(\cdot)+\int_{0}^{\cdot} Y_{N}(u) \mathrm{d} u, t\right)
$$

(see page 658, line 4 of [5]). Firstly, it is not clear how to understand the words 'up to $\tau_{N}^{M_{Y}}$, since $\tau_{N}^{M_{Y}}$ is defined through $Y$ in the formulation of Proposition 1, while $Y$ is still undefined. Secondly, this statement is incorrect because (4) is just an identity that holds for the particular processes $X_{N}$ and $W^{\mathrm{Q}_{N}}$, while (5) is an equation in $Y_{N}$, where $W$ is the given initial Brownian motion under P. Using an argument similar to this one in [5], one can conclude that the existence of a weak solution of a stochastic differential equation 'demonstrates the existence of' a strong solution of the same equation, which, however, is false, as is well known; see, e.g. [1, Chapter 5, Exercise 3.5] or item 6 of this section. Furthermore, even if this transition from (4) to (5) were 
in order, one would not be able to take limits as $N \rightarrow \infty$ as suggested in [5, p. 658, line 6] because nothing is said about the uniqueness of $Y_{N}$ satisfying (5) nor about the consistency properties of the 'solutions' $Y_{N}$ of (5) for different $N$. One must conclude that the proof of Proposition 1 in [5] is invalid, in whichever way one interprets the statement.

This in turn invalidates the main result: Theorem 1 on page 658 of [5] is misleading since its formulation and proof use the process $Y$ from Proposition 1 of [5].

2. In Definition 1 on page 660 of [5], which we now quote, the authors 'defined' the measure $\mathrm{Q}^{C}$ as follows.

Definition 1. (Definition 1 of [5].) A candidate measure $\mathrm{Q}^{C}$, corresponding to the process $X(t)$ defined in Proposition 1 on the measure $\mathrm{P}$, is a measure corresponding to which

$$
X(t)=\xi\left(W^{\mathrm{Q}^{C}}(\cdot)+\int_{0}^{\cdot} X(u) \mathrm{d} u, t\right)
$$

is defined (possibly up to the explosion time $\tau^{M_{X}}$ ), with $W^{\mathrm{Q}^{C}}$ a $\mathrm{Q}^{C}$-Brownian motion.

This 'definition' is unclear, regarding both existence (of $\mathrm{Q}^{C}$ and $W^{\mathrm{Q}^{C}}$ ) and uniqueness. In [5] the authors stated that $\mathrm{Q}^{C}$ is well defined by the analysis in Proposition 1, but this argument is invalid as discussed above. Indeed, counterexamples in items 1 and 2 of Section 4 below show that both existence and uniqueness of $\mathrm{Q}^{C}$ 'defined' in this way may fail. This in turn invalidates their next result, Corollary 1 , on page 660 of [5].

3. We now turn our attention to Corollary 2 on page 661 of [5], which the authors formulated as follows.

Corollary 1. (Corollary 2 of [5].) Assume that $X(t)$ is the unique weak solution up to the explosion time $\eta_{X}$ of the functional stochastic differential equation (SDE)

$$
\mathrm{d} X(t)=\mu(X, t) \mathrm{d} t+\sigma(X, t) \cdot \mathrm{d} W(t)
$$

with initial value $X(0)$ and $\mu(x, t) \in \mathbb{R}^{d}, \sigma(x, t) \in \mathbb{R}^{d \times r}$, with $\mu(x, t), \sigma(x, t)$ progressively measurable functionals. Then

$$
\mathrm{E}_{\mathrm{P}}\left[Z_{X}(T)\right]=\mathrm{Q}^{C}\left(\eta^{X}>T\right)
$$

where

$$
\mathrm{d} X(t)=(\mu(X, t)+\sigma(X, t) \cdot X(t)) \mathrm{d} t+\sigma(X, t) \cdot \mathrm{d} W^{\mathrm{Q}^{C}}(t) .
$$

The first minor point here is that $X$ is $d$-dimensional and $W$ is $r$-dimensional, while it is important in the definition of the process $Z_{X}$ that $X$ and $W$ have the same dimension.

The explosion time $\eta^{X}$, which appears in Corollary 2 of [5], is defined in the last paragraph on page 660 of [5] by

$$
\eta^{X}=\lim _{N \rightarrow \infty} \eta_{N}^{X}, \quad \text { where } \quad \eta_{N}^{X}=\inf \left\{t \in[0, \infty): \sup _{i=1, \ldots, d}\left|X_{i}(t)\right| \geq N\right\}
$$

and the $X_{i}(t), i=1, \ldots, d$, are components of $X(t)$. Let us add at this point that both $\tau^{M_{X}}$ and $\eta^{X}$ are termed 'the explosion time' in [5] (see, e.g. the above formulations of Proposition 1 and Definition 1 quoted from [5]), which is confusing because these stopping times can be different even in the setting of Corollary 2 of [5] (e.g. take an appropriate deterministic $X$ ). In our paper only $\eta^{X}$ is called 'the explosion time' with the exception of the statements that we quote from [5]. 
We conclude that Corollary 2 of [5] is also invalid, as it is unclear what the measure $\mathrm{Q}^{C}$ represents.

4. We now seek a well-posed reformulation of Corollary 2 of [5]. There is a natural candidate, as follows (though this is still incorrect, as we show next). Since $W^{\mathrm{Q}^{C}}$ is assumed to be a $\mathrm{Q}^{C}$ Brownian motion and SDE (9) is announced to hold, it is natural to suggest the following.

Corollary $2 *$. Let X be a unique in law possibly explosive weak solution of the SDE

$$
\mathrm{d} X(t)=\mu(X, t) \mathrm{d} t+\sigma(X, t) \cdot \mathrm{d} W(t)
$$

on some filtered probability space $\left(\Omega, \mathcal{F},\left(\mathcal{F}_{t}\right), \mathrm{P}\right)$ with initial value $X(0)$ and $\mu(x, t) \in \mathbb{R}^{d}$, $\sigma(x, t) \in \mathbb{R}^{d \times d}$, where $\mu(x, t), \sigma(x, t)$ are progressively measurable functionals. Consider the process

$$
Z_{X}(t)=\exp \left\{\int_{0}^{t \wedge \eta^{X}} X(u) \cdot \mathrm{d} W(u)-\frac{1}{2} \int_{0}^{t \wedge \eta^{X}}\|X(u)\|^{2} \mathrm{~d} u\right\} \text { for } t \in[0, \infty),
$$

where we set $Z_{X}(t)=0$ for $t \geq \eta^{X}$ on the event $\left\{\int_{0}^{\eta^{X}}\|X(u)\|^{2} \mathrm{~d} u=\infty\right\}$. Assume further that $\tilde{X}$ is a unique in law possibly explosive weak solution of the SDE

$$
\mathrm{d} \tilde{X}(t)=(\mu(\tilde{X}, t)+\sigma(\tilde{X}, t) \cdot \tilde{X}(t)) \mathrm{d} t+\sigma(\tilde{X}, t) \cdot \mathrm{d} \tilde{W}(t)
$$

on some filtered probability space $(\tilde{\Omega}, \tilde{\mathcal{F}},(\tilde{\mathcal{F}}), \tilde{\mathrm{P}})$ with the same initial value $X(0)$. Then

$$
\mathrm{E}_{\mathrm{P}}\left[Z_{X}(T)\right]=\tilde{\mathrm{P}}\left(\eta^{\tilde{X}}>T\right),
$$

where $\eta^{\tilde{X}}$ is the explosion time of $\tilde{X}$.

Let us point out the difference between Corollary 2 of [5] and Corollary $2 *$. In the latter the existence of a unique in law weak solution is assumed for each SDE separately, possibly on different probability spaces, while in the former both measures $\mathrm{P}$ and $\mathrm{Q}^{C}$ are stated to be on the same space and the process $X$ is claimed to solve the two SDEs under the two measures, respectively.

5. Before we proceed with Corollary $2 *$ let us point out a further inconsistency in the formulation of Corollary 2 of [5], which is also fixed in the formulation of Corollary $2 *$. In the formulation of Corollary 2 of [5] the authors go beyond their setting. Namely, allowing $X$ to be explosive is inconsistent with their definition of $\tau^{M_{X}}$ and their stipulation that the process $X$ is stopped at $\tau^{M_{X}}$. This is particularly relevant if, e.g. we have

$$
\eta^{X}<\infty \quad \text { P-a.s. } \quad \text { and } \quad \int_{0}^{\eta^{X}}\|X(u)\|^{2} \mathrm{~d} u<\infty \quad \text { P-a.s. }
$$

since in this case (2) does not define $\tau^{M_{X}}$ (unless it is specified what $X$ is after $\eta^{X}$; note that

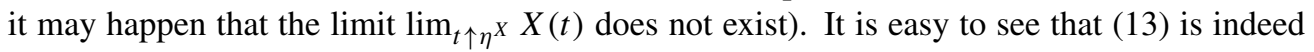
possible (take, e.g. an appropriate deterministic $X$, which corresponds to the zero matrix $\sigma$ in (7); in item 4 of Section 4 we also give a stochastic example where (13) holds). Thus, if (13) holds and the behaviour of $X$ after $\eta^{X}$ is not specified (which is the case in the setting of Corollary 2 of [5]), then $\tau^{M_{X}}$ is undefined, $Z_{X}$ is also undefined (the authors of [5] defined $Z_{X}$ via (3); see Section 2 of [5]), and, hence, the left-hand side of (8) is undefined as well. 
In the proof of Wong and Heyde's Corollary 2 on page 661 of [5] they claimed that $\eta^{X}=\tau^{M_{X}}$ a.s., which is incorrect as we have just seen that $\tau^{M_{X}}$ may be undefined in the setting of their Corollary 2. (In the formula in lines 2 and 3 of the proof of Corollary 2 in [5] it is stated that $\eta^{X}$ and $\tau^{M_{X}}$ have the same law (note that the mentioned formula is claimed to hold for any $T$ ). However, since it is clear that $\eta^{X} \leq \tau^{M_{X}}$ a.s. (provided it is specified what $X$ is after $\eta^{X}$, to be able to speak about $\tau^{M_{X}}$ ), this claim amounts to $\eta^{X}=\tau^{M_{X}}$ a.s.) Moreover, even if the behaviour of $X$ after $\eta^{X}$ were specified (so that the $\tau^{M_{X}}$ were well defined) then the claim that $\eta^{X}=\tau^{M_{X}}$ a.s. would also be incorrect (e.g. if (13) holds and we specify $X(t)=0$ for $t \geq \eta^{X}$, then $\tau^{M_{X}}=\infty>\eta^{X}$, P-a.s.). In order to define $Z_{X}$ in the setting of Corollary 2 of [5] we need to use (11), in which case no problems arise and the behaviour of $X$ after $\eta^{X}$ is not essential at all.

6. Furthermore, it should be emphasised that Corollary 2, as stated in [5], goes beyond the setting of [5] in another respect also, and, hence, even if there were no issues with Proposition 1 and Definition 1, and the other issues with Corollary 2 of [5] discussed above did not exist, it could not be proved as claimed in [5]. Recall that a solution (or a weak solution) of the SDE

$$
\mathrm{d} X(t)=\mu(X, t) \mathrm{d} t+\sigma(X, t) \mathrm{d} W(t), \quad X(0)=x_{0},
$$

is a pair of adapted processes $(X, W)$ on some filtered probability space $\left(\Omega, \mathcal{F},\left(\mathscr{F}_{t}\right), \mathrm{P}\right)$ such that $W$ is an $\left(\mathscr{F}_{t}\right)$-Brownian motion,

$$
\int_{0}^{t}\left(|\mu(X, u)|+\sigma^{2}(X, u)\right) \mathrm{d} u<\infty \quad \text { P-a.s., } t \in[0, \infty),
$$

and

$$
X(t)=x_{0}+\int_{0}^{t} \mu(X, u) \mathrm{d} u+\int_{0}^{t} \sigma(X, u) \mathrm{d} W(u) \quad \text { P-a.s., } t \in[0, \infty) .
$$

(For notational simplicity, we consider only one-dimensional $X$ and $W$, and define only a nonexplosive solution here because this is all that we need for the argument with Tanaka's SDE below.) Note that the filtered probability space $\left(\Omega, \mathcal{F},\left(\mathscr{F}_{t}\right), \mathrm{P}\right)$ in this definition may differ from that described in Section 2. A strong solution $(X, W)$ of this SDE is a solution such that the process $X$ is adapted to the filtration generated by the Brownian motion $W$ (see [4, Chapter IX, Section 1] for the employed terminology). It is easy to show that Tanaka's SDE

$$
\mathrm{d} X(t)=\operatorname{sgn} X(t) \mathrm{d} W(t), \quad X_{0}=0,
$$

where

$$
\operatorname{sgn} x= \begin{cases}1 & \text { if } x>0 \\ -1 & \text { if } x \leq 0\end{cases}
$$

has a unique in law weak solution (and, moreover, $X$ is a Brownian motion by Lévy's characterisation theorem for any solution $(X, W)$ of (14)). However, there exists no strong solution of (14): for any solution $(X, W)$, we have

$$
W(t)=\int_{0}^{t} \operatorname{sgn} X(u) \mathrm{d} X(u) \quad \text { P-a.s. for all } t \geq 0,
$$

and, hence, by [4, Chapter VI, Corollary 2.2], the filtration generated by $W$ coincides with that generated by $|X|$, which is strictly smaller than the filtration generated by $X$, as $X$ is a Brownian 
motion. Thus, $X$ cannot be adapted to the filtration generated by $W$. This argument implies that a solution of Tanaka's SDE cannot be expressed as $X(t)=\xi(W(\cdot), t)$ for a progressively measurable functional $\xi$, and, hence, does not satisfy the assumptions of Proposition 1 and Theorem 1 of [5]. To summarize the last point, even if all other results in [5] were beyond reproach, the weak existence, which is assumed in their Corollary 2, would be an insufficient assumption to support the conclusions of Corollary 2 using their method. The authors of [5] should have assumed existence of a strong solution of (7) in Corollary 2.

7. The discussion above leads to the question of whether Corollary $2 *$ holds. Or, at least, whether such a statement holds under the stronger assumptions that $X$ and $\tilde{X}$ are pathwise unique strong solutions of SDEs (10) and (12). A counterexample in item 3 of Section 4 shows that the answer is negative. Moreover, $X$ and $\tilde{X}$ are pathwise unique strong solutions of those SDEs in that counterexample.

\section{Counterexamples}

1. We start with two counterexamples to Definition 1 of [5]. Let us take $d=1$, fix a finite time horizon $T \in(0, \infty)$, and consider $\Omega=C([0, T], \mathbb{R})$, the space of continuous functions $[0, T] \rightarrow \mathbb{R}$. Let $W$ be the coordinate process on $\Omega$, let P be the Wiener measure, let $\left(\mathcal{F}_{t}\right)_{t \in[0, T]}$ be the filtration generated by $W$ and augmented to satisfy the usual conditions, and let $\mathcal{F}=\mathscr{F}_{T}$, so that we are in the setting of [5].

First we show that the measure $\mathrm{Q}^{C}$ in Definition 1 of [5] may not be unique. Indeed, take $\xi(\cdot, \cdot) \equiv 0$, so that $X \equiv 0$ as well (recall that $X$ is defined by the formula $X(t)=\xi(W(\cdot), t)$ ). Note that, for any $\lambda \in \mathbb{R}$, the process

$$
W^{\lambda}(t)=W(t)-\lambda t, \quad t \in[0, T]
$$

is an $\left(\mathcal{F}_{t}, \mathrm{P}^{\lambda}\right)$-Brownian motion, where the measure $\mathrm{P}^{\lambda}$ is given by

$$
\frac{\mathrm{dP}^{\lambda}}{\mathrm{dP}}=\exp \left\{\lambda W(T)-\frac{\lambda^{2}}{2} T\right\} .
$$

Clearly, any measure $\mathrm{P}^{\lambda}$ (and, in fact, many other measures) can be considered as $\mathrm{Q}^{C}$ because (6) is satisfied with $W^{\mathrm{Q}^{C}}=W^{\lambda}$, which is a $\mathrm{P}^{\lambda}$-Brownian motion, as required in Definition 1 of [5].

2. Now we show that the measure $\mathrm{Q}^{C}$ in Definition 1 of [5] may not exist. We consider the filtered probability space as above and a strictly increasing continuous function $f: \mathbb{R} \rightarrow \mathbb{R}$ with

$$
\lim _{x \rightarrow-\infty} f(x)=1 \text { and } \lim _{x \rightarrow \infty} f(x)=2 .
$$

Let us define a progressively measurable functional $\xi(\omega, t), \omega \in \Omega(=C([0, T], \mathbb{R})), t \in$ $[0, T]$, by the formula

$$
\xi(\omega, t)= \begin{cases}\frac{f(\omega(t))}{T-t} & \text { if } t \in[0, T), \\ 0 & \text { if } t=T,\end{cases}
$$

which gives us the process $X(t)=\xi(W(\cdot), t), t \in[0, T]$.

Let us prove that there exists no measure $\mathrm{Q}^{C}$ satisfying Definition 1 of [5]. Since $X(t)=$ $f(W(t)) /(T-t), t \in[0, T)$, and $f$ is strictly increasing, trajectories of $X$ determine trajectories 
of $W$ uniquely. In particular, if (6) holds, the process $W^{\mathrm{Q}^{C}}$ should satisfy

$$
W^{\mathrm{Q}^{C}}(t)=W(t)-\int_{0}^{t} X(u) \mathrm{d} u, \quad t \in[0, T) .
$$

It follows that

$$
\lim _{t \uparrow T} W^{\mathrm{Q}^{C}}(t)=-\infty \quad \text { for any } \omega \in \Omega
$$

(recall (15) and (16), and note that $W(t) \rightarrow W(T) \in \mathbb{R}$ as $t \uparrow T$ for any $\omega \in \Omega$ because $W$ is the coordinate process on the space of continuous functions); hence, there does not exist a measure $\mathrm{Q}^{C}$ on $(\Omega, \mathcal{F})$ such that $W^{\mathrm{Q}^{C}}$ is a $\mathrm{Q}^{C}$-Brownian motion.

3. We proceed with a counterexample to Corollary $2^{*}$. Let $\mu(x)=|x|^{\alpha}$ for any fixed $\alpha>3$, and let $\sigma(x) \equiv 1$. The process $X(t)$, with the state space $\mathbb{R}$ and starting value $X(0) \in \mathbb{R}$, can be defined as a strong solution of the SDE

$$
\mathrm{d} X(t)=|X(t)|^{\alpha} \mathrm{d} t+\mathrm{d} W(t)
$$

up to the explosion time $\eta^{X}$. The existence of a strong solution up to $\eta^{X}$ and pathwise uniqueness are guaranteed by Itô's existence and uniqueness theorem since the coefficients of the SDE are locally Lipschitz (see [4, Chapter IX, Exercise 2.10]). It follows from Example 3.1 of [3] that the process

$$
Z_{X}(t)=\exp \left\{\int_{0}^{t \wedge \eta^{X}} X(u) \mathrm{d} W(u)-\frac{1}{2} \int_{0}^{t \wedge \eta^{X}} X^{2}(u) \mathrm{d} u\right\}, \quad t \in[0, \infty)
$$

$\left(Z_{X}(t)=0\right.$ for $t \geq \eta^{X}$ on $\left\{\int_{0}^{\eta^{X}} X^{2}(u) \mathrm{d} u=\infty\right\}$; see, however, (19) below), is a martingale (in fact, it is even a uniformly integrable martingale). Hence, we have

$$
\mathrm{E}_{\mathrm{P}}\left[Z_{X}(T)\right]=1 \quad \text { for all } T \geq 0 .
$$

In this case, $\operatorname{SDE}(12)$ has the form

$$
\mathrm{d} \tilde{X}(t)=\left(|\tilde{X}(t)|^{\alpha}+\tilde{X}(t)\right) \mathrm{d} t+\mathrm{d} \tilde{W}(t) .
$$

Its coefficients are locally Lipschitz and, therefore, there exists a pathwise unique strong solution up to the explosion time $\eta^{\tilde{X}}$. The process $\tilde{X}$ explodes to $+\infty$ in finite time, which follows from Feller's test for explosions (see [1, Chapter 5, Theorem 5.29 and Proposition 5.32]). Thus,

$$
\tilde{\mathrm{P}}\left(\eta^{\tilde{X}}>T\right)<1 \quad \text { for sufficiently large } T>0,
$$

which now contradicts the claim in Corollary $2 *$.

Note that since SDE (18) has a pathwise unique strong solution, we can construct a solution of this SDE on the same probability space that supports the solution of SDE (17) with the same Brownian motion $W$ as in (17). This means that the reason why Corollary $2 *$ does not hold is not due to the fact that the solutions of SDEs (10) and (12) are allowed to exist on distinct probability spaces; in fact, this reason is deeper. See [3] for more details on this point.

4. Finally, as promised in Section 3, we demonstrate that (13) is possible. Namely, (13) holds in the example in item 3 of this section. Indeed, by Feller's test for explosions, P-almost all trajectories of $X$ explode at $+\infty$, and, hence, $\eta^{X}<\infty$, P-a.s. We now need to prove that

$$
\int_{0}^{\eta^{X}} X_{s}^{2} \mathrm{~d} s<\infty \quad \text { P-a.s. }
$$


in this example. Property (19) is equivalent to $Z_{X}\left(\eta^{X}\right)>0$, P-a.s., which is in turn equivalent to the property that

$$
Z_{X}(\infty)>0 \quad \text { P-a.s. }
$$

(note that $Z_{X}$ is stopped at $\eta^{X}$ ). It remains to note that (20) holds in the case when the process $X$ is given by (17) (with $\alpha>3$ as above), which follows from Theorem 2.2 of [2]. Namely, condition (II) in Theorem 2.2 of [2] is satisfied.

\section{References}

[1] Karatzas, I. And Shreve, S. E. (1991). Brownian Motion and Stochastic Calculus (Graduate Texts Math. 113), 2nd edn. Springer, New York.

[2] Misatović, A. And Urusov, M. (2010). Deterministic criteria for the absence of arbitrage in one-dimensional diffusion models. To appear in Finance Stoch.

[3] Misatović, A. ANd Urusov, M. (2010). On the martingale property of certain local martingales. To appear in Prob. Theory Relat. Fields.

[4] Revuz, D. And Yor, M. (1999). Continuous Martingales and Brownian Motion (Fundamental Principles Math. Sci. 293), 3rd edn. Springer, Berlin.

[5] Wong, B. And Heyde, C. C. (2004). On the martingale property of stochastic exponentials. J. Appl. Prob. 41, 654-664. 